\title{
ON THE CHARACTERIZATION OF LINEAR AND PRO JECTIVE LINEAR GROUPS. II
}

\author{
BY \\ JOHN H. WALTER(1)

\section{Introduction ANd Preliminaries}

1. Introduction. This is a sequel to an earlier paper, On the characterization of linear and projective linear groups. I, [4]. Its purpose is to characterize a class of groups $B$ such that $P T L(n, K) \subseteq B \subseteq P G L(n, K)$. Here $P G L(n, K)$ is the projective general linear group, that is, the group of projectivities of a projective geometry formed from an $n$-dimensional vector space over a field $K$. This is isomorphic to the factor group of the general linear group $G L(n, K)$ by its center. By the group $\left({ }^{2}\right) T L(n, K)$, we mean the normal subgroup of $G L(n, K)$ generated by its involutions $\left({ }^{(}\right) ; P T L(n, K)$ designates the image of this group in $P G L(n, K)$ under the natural homomorphism of $G L(n, K)$. It contains the image $P S L(n, K)$ of the unimodular group as a subgroup of index at most 2. Using the terminology of [4], we term groups such as (S) as projective linear groups of rank $n$. The characterization is obtained for $n \geqq 4$ and $K$ commutative $\left({ }^{4}\right)$ of characteristic not 2 by introducing a class of groups to be called quasiprojective. Roughly speaking, a quasiprojective group is described by specifying the centralizers of its involutions and $a$ few other conditions related to the center and the factor commutator group. The centralizers of involutions are described in terms of the quasilinear groups introduced in [4]. Quasilinear groups were defined as prototypes of groups $(B)$ such that $T I(n, K) \subseteq B \subseteq G L(n, K)$, which are called linear groups of rank $n$, in much the same manner that quasiprojective groups are to be defined as prototypes of projective linear groups. The principal theorem of the first paper characterized the factor group of a quasilinear group by its center as a projective linear group. This paper carries out the techniques and results of the first paper to their ultimate conclusion, namely, a direct characterization of the projective linear groups.

Because the techniques and results of this paper are so closely related to those of the first paper, we will treat it as a continuation and will presume that the reader is familiar with the definitions, notation, and results of that

Received by the editors September 2, 1961.

(1) The author was supported by the National Science Foundation during the preparation of this paper (grant G-11098).

(2) In [4], we used the notation $T L(n), \operatorname{PTL}(n)$, etc.

(3) An involution is understood to be an element of order 1 or 2 . The identity is called the trivial involution.

(4) In [4], we required only that $K$ be a division ring of characteristic not 2. 
paper. To refer to a proposition, formula, or other enumerated reference of [4], we prefix the symbol I as in Proposition I.8.1.

2. Fused products. In order to define a quasiprojective group, we introduce the concept of a fused product $\mathfrak{B}$ of two subgroups $\mathfrak{A}$ and $\mathfrak{B}$, which we will write as $\mathfrak{B}=\mathfrak{A} \circ \mathfrak{B}$. Here we demand that $\mathfrak{A}$ and $\mathfrak{B}$ be normal subgroups such that $\mathfrak{B}=\mathscr{P} \mathfrak{B}, \mathfrak{A} \subseteq C(\mathscr{B})$ and that

$$
\mathscr{A} \cap \mathfrak{B} \subseteq Z(\mathfrak{A}) \cap Z(\mathfrak{B}) .
$$

A fused product for which $\mathscr{A} \cap \mathscr{P}=Z(\mathfrak{A})=Z(\mathfrak{B})=Z(\mathfrak{H} \circ \mathfrak{B})$ will be called a maximal fused product (sometimes called a central product).

If $C \in \mathscr{P} \circ \mathfrak{B}$ and $C=A B$ where $A \in \mathscr{P}$ and $B \in \mathfrak{B}$, we will write $C=A \circ B$ and call this a representation of $C$ relative to the decomposition $\mathfrak{B}=\mathfrak{U} \circ \mathfrak{V}$. Of course, the elements $A$ and $B$ are not uniquely determined. In fact, we may have $C=A^{\prime} \circ B^{\prime}$ as long as $A A^{\prime-1}=B B^{\prime-1}$ is in $\mathscr{A} \cap \mathscr{B}$.

Given a direct product $\mathscr{A} \times \mathfrak{B}$ of groups $\mathscr{A}$ and $\mathfrak{B}$, let $f$ be a homomorphism of $\mathfrak{A} \times \mathfrak{B}$ with kernel $\mathfrak{D}$ such that $\mathfrak{A} \cap \mathfrak{D}=\mathfrak{B} \cap \mathfrak{D}=1$ and $\mathfrak{D} \subseteq Z(\mathfrak{A}) \times Z(\mathfrak{B})$. It is easy to see that $f(\mathscr{A} \times \mathfrak{B})$ is the fused product $f(\mathfrak{Q}) \circ f(\mathfrak{B})$ of the images of $\mathfrak{A}$ and $\mathfrak{B}$.

3. Projective linear groups. To motivate our definition of quasiprojective groups, we will review the description of the centralizers of involutions in the group $P G L(n, K)$ given in Dieudonné's treatise, $[2,8 \delta 3,4]$.

The elements $\bar{U}$ of $G L(n, K)$ which map onto involutions under the canonical homomorphism $\Lambda$ of $G L(n, K)$ onto $P G L(n, K)$ are called projective involutions. They are characterized by the fact that $\bar{U}^{2}$ is in the center of $G L(n, K)$. We term a projective involution $\vec{U}$ and its image $U=\Lambda \vec{U}$ to be of the first kind if $\bar{U}^{2}$ is a square in the center 3 of $G L(n, K)$. If $\bar{U}^{2}$ is not a square in $\overline{3}$, we term $U$ and $\bar{U}$ to be of the second kind. If $\bar{U}$ is a projective involution of the first kind, there is an involution in the coset $\bar{U} \overline{3}$. Because of this, we will always be able to consider that $\bar{U}$ is actually an involution in $G L(n, K)$ if $\bar{U}$ is of the first kind.

The centralizer $C_{\bar{\Theta}}(\bar{U})$ of an involution $\bar{U}$ in $\bar{\Theta}=G L(n, K)$ is the direct product of full linear groups $\overline{\mathfrak{u}}_{1}$ and $\overline{\mathfrak{U}}_{2}$ such that rk $\overline{\mathfrak{u}}_{1}+$ rk $\overline{\mathfrak{U}}_{2}=n$. The conjugate classes of involutions in $\bar{\Theta}, \bar{\Omega}_{0}, \bar{\Omega}_{1}, \cdots, \bar{\Omega}_{n}$, may be so enumerated(") that $\bar{U} \in \bar{\Omega}_{p}$ implies $C_{\overline{\mathbb{G}}}(U)=\overline{\mathfrak{U}}_{1} \times \overline{\mathfrak{U}}_{2}$ and rk $\overline{\mathfrak{U}}_{1}=p$.

The elements of $G L(n, K)$ which map onto the centralizer of an involution $\Lambda \bar{U}$ in $P G L(n, K)$ are the elements which commute or anticommute( $\left.{ }^{\circ}\right)$. This means that there is a subgroup $C_{1}(\bar{U})$ of $G L(n, K)$ which contains the centralizer $C_{\bar{\Theta}}(\bar{U})$ as a subgroup of index at most 2 . The index is 2 when there exists an element $\bar{W}$ anticommuting with $\bar{U}$, and this can happen only if $\mathrm{rk} \overline{\mathfrak{u}}_{1}=\mathrm{rk} \overline{\mathfrak{u}}_{2}=n / 2$. The anticommuting element may be taken to be an in-

(5) A quasilinear or linear group of rank 0 is understood to be the identity group.

( ${ }^{\circ}$ An element $A \in \Theta$ anticommutes with an element $B$ if $A B=-B A$. 
volution and $\bar{W} \bar{U}_{1} \bar{W}^{-1}=\bar{U}_{2}$. We will use the notation $\mathscr{Q} \times{ }_{2} \mathscr{B}$ to denote a semidirect product $\left({ }^{7}\right)$ of two groups. Thus we have that

$$
C_{1}(\overline{\mathfrak{u}})=\overline{\mathfrak{B}} \times{ }_{\mathfrak{s}}\left(\overline{\mathfrak{U}}_{1} \times \overline{\mathfrak{U}}_{2}\right)
$$

where $\mathfrak{B}$ is a subgroup of order 1 or 2 according as $\bar{U}$ is not or is in $\bar{\nexists}_{n / 2}$.

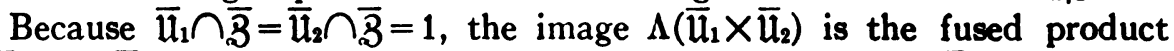
$\Lambda\left(\overline{\mathfrak{U}}_{1}\right) \circ \Lambda\left(\overline{\mathfrak{U}}_{2}\right)$. In this case, to every element $\bar{X}=\bar{X} \times 1$ in $Z\left(\overline{\mathfrak{U}}_{1}\right)$, there corresponds an element $\bar{X} \times \bar{X}^{\prime}$ in $\bar{Z}$. Then $\Lambda(\bar{X})=\Lambda\left(\bar{X}^{\prime-1}\right)$. Thus $\Lambda Z\left(\overline{\mathfrak{U}}_{1}\right)$ $\subseteq \Lambda Z\left(\overline{\mathfrak{U}}_{2}\right)$; and, by symmetry, $\Lambda Z\left(\overline{\mathfrak{u}}_{1}\right)=\Lambda Z\left(\overline{\mathfrak{U}}_{2}\right)$. As $\overline{\mathfrak{u}}_{1} \cap \overline{\mathrm{B}}=\overline{\mathfrak{u}}_{2} \cap \overline{\bar{B}}=1$, $Z\left(\mathfrak{U}_{1}\right)=\Lambda Z\left(\overline{\mathfrak{u}}_{1}\right)=\Lambda Z\left(\overline{\mathfrak{u}}_{2}\right)=Z\left(\mathfrak{U}_{2}\right)$ where $\mathfrak{u}_{1}=\Lambda \overline{\mathfrak{u}}_{1}$ and $\mathfrak{U}_{2}=\Lambda \overline{\mathfrak{U}}_{2}$. Thus $\Lambda\left(\overline{\mathfrak{u}}_{1} \times \overline{\mathfrak{u}}_{2}\right)$ is a maximal fused product $\mathfrak{u}_{1} \circ \mathfrak{U}_{2}$.

Corresponding to the $n+1$ classes of involutions in $G L(n, K)$, there are $[n / 2]+1$ classes of involutions $\mathfrak{R}_{0}, \mathfrak{R}_{1}, \ldots, \mathfrak{R}_{[n / 2]}$ where $\mathfrak{R}_{n}=\Lambda \bar{\Re}_{p}=\Lambda \mathbb{\Omega}_{n-p}$. It will be convenient to set $\ell_{p}=\ell_{n-p}$ for $[n / 2] \leqq p \leqq n$. Hence for $U \in \ell_{p}$

$$
C_{P(\bar{\Theta})}(U)=\mathfrak{B} \times_{\mathbf{1}}\left(\mathfrak{U}_{1} \circ \mathfrak{U}_{2}\right)
$$

where $\mathfrak{U}_{1}$ and $\mathfrak{U}_{2}$ are full linear groups of ranks $p$ and $n-p, \mathfrak{u}_{1} \circ \mathfrak{U}_{2}$ is a maximal fused product, $\mathfrak{B}$ is of order 1 or 2 and of order 2 only if $p=n / 2$, in which case $W \mathfrak{u}_{1} W^{-1}=\mathfrak{u}_{2}$.

If $\bar{U}$ is a projective involution of the second kind in $G L(n, K)$, then $n$ is even and $C(\bar{U})$ is isomorphic to $G L(n / 2, L)$ where $L$ is a quadratic extension of $K$. In particular, $C(\bar{U})$ contains the group $G L(n / 2, L)$ as a subgroup of index 2 . It is important to note that only those involutions in $\Omega_{p}$ with $p$ even commute with $\bar{U}$. The centralizer of the image $\Lambda \bar{U}$ of $\bar{U}$ in $P G L(n, K)$ then contains a factor group of $G L(n / 2, L)$ by a subgroup of its center as a subgroup of index at most 2 . Only involutions in the classes $\ell_{p}$ with $p$ even commute with involutions of the second kind.

The classes of involutions in $T L(n, K)$ and $\operatorname{PTL}(n, K)$ are also the classes of involutions in $G L(n, K)$ and $P G L(n, K)$, respectively.

4. Quasiprojective groups. We define a quasiprojective group of rank $n \geqq 4$ to be a group (s) satisfying the following axioms.

Aхгом A. The group \&5 possesses $[n / 2]$ classes of involutions $\mathfrak{R}_{1}, \mathfrak{R}_{2}, \cdots$, $\mathfrak{R}_{[n / 2]}$ such that for $U \in \mathfrak{R}_{p}$, the centralizer $C(U)$ is a subgroup of the semidirect product $C^{\prime \prime}(U)$

$$
C^{\prime \prime}(U)=\mathfrak{B} \times_{\bullet}\left(\mathfrak{U}_{1} \circ \mathfrak{U}_{2}\right)
$$

of a group $\mathfrak{B}$ of order 1 or 2 with a maximal fused product of full quasilinear groups $\mathfrak{U}_{1}$ and $\mathfrak{U}_{2}$ of ranks $p$ and $n-p$ which contains the fused product

$$
C^{\prime}(U)=T\left(\mathfrak{U}_{1}\right) \circ T\left(\mathfrak{U}_{2}\right)
$$

( ${ }^{7}$ A semidirect product $\mathfrak{U} \times{ }_{\mathfrak{B}} \mathfrak{B}$ of two groups $\mathfrak{A}$ and $\mathfrak{B}$ is a group which is a product $\mathfrak{A} \mathscr{B}$ of a group $\mathscr{A}$ and a normal subgroup $\mathfrak{B}$. (Cf. [3, pp. 88].) 
of the involutory subgroups of $\mathfrak{U}_{1}$ and $\mathfrak{U}_{2} . \mathfrak{B}$ is of order 2 only if $p=n / 2$. Then if $W$ is the involution in $\mathfrak{B}, W \mathfrak{u}_{1} W^{-1}=\mathfrak{U}_{2}$.

The identity is in the class $\&_{0}$. Only involutions in the classes $\mathfrak{R}_{p}, 0 \leqq p \leqq[n / 2]$ commute with those in the class $\mathfrak{R}_{1}$.

The involutions of the classes $\ell_{p}, 0 \leqq p \leqq[n / 2]$, will be called involutions of the first kind. For convenience, we will set $\ell_{p}=\Omega_{n-p}$ for $[n / 2] \leqq p \leqq n$. We will call the involutions in the classes $\mathbb{R}_{p} p$-involutions of $\mathbb{S}$.

Aхгом B. The center of $\&$ consists of the identity alone. The centralizer of the group generated by the involutions in the classes $\mathfrak{R}_{p}$ also consists of the identity.

Axıм $C$. When $B$ is an infinite group, $U \in \mathfrak{R}_{1}$, and $V \in \mathfrak{R}_{p}, p>1$, then there exists an involution $W \neq 1$ of the first kind in $C(U, V)$.

The Principal Theorem of [4] (hereinafter denoted as Principal Theorem I) asserts that if $\mathfrak{G}$ is a quasilinear group of rank $n, P(\mathfrak{W})$ is a projective linear group of rank $n$. We will use this result often. In this paper we will prove:

PRINCIPAL THEOREM II. Let \& be a quasiprojective group of rank $n \geqq 4$ if \& is finite and of rank $n \geqq 5$ if \&s is infinite. Then \&s is a projective linear group of rank $n$.

For the remainder of this paper, \& will represent a quasiprojective group of rank $n \geqq 4$ for which we will prove this theorem.

\section{Characterizations of Lower Centralizers}

By the upper centralizer of an involution $U$ of the first kind in a quasiprojective group $G$, we mean the group $C^{\prime \prime}(U)$ described in (4.1). By the lower centralizer of an involution of the first kind, we mean the subgroup $C^{\prime}(U)$ described in (4.2). We wish to identify a certain set of involutions as generators of the lower centralizer of an involution in order to allow us to treat lower centralizers in the manner in which we treated involutory centralizers in [4].

5. Involutions in fused products. Suppose that $\mathfrak{B}$ is the fused product of quasilinear groups $\mathfrak{B}=\mathfrak{U} \circ \mathfrak{B}$. Let $R$ be an involution in $\mathfrak{B}$ so that $R=S \circ T$ relative to this decomposition. Then $R^{2}=1=S^{2} \circ T^{2}$. Hence $A=S^{2}=T^{-2}$ is an element of $\mathfrak{u} \cap \mathfrak{B} \subseteq Z(\mathfrak{U} \circ \mathfrak{B})$. If $R=S^{\prime} \circ T^{\prime}$ is a second representation of $R$, then we have seen that $S^{\prime}=S Z$ and $T^{\prime}=T Z^{-1}$ with $Z \in Z(\mathfrak{U} \circ \mathfrak{B})$. Then $S^{\prime 2}=A Z^{2}$ and $T^{\prime 2}=\left(A Z^{2}\right)^{-1}$. We say that $R$ is a regular or irregular involution in $\mathfrak{U} \circ \mathfrak{B}$ according as $A$ is a square in $Z(\mathfrak{U} \circ \mathfrak{B})$ or not. If $R$ is in the centralizer $C(U)$ of an involution of the first kind, then we say that $R$ is regular in $C(U)$ or $C^{\prime \prime}(U)$ if it belongs to the fused product $\mathfrak{U}_{1} \circ \mathfrak{l}_{2}$ of (4.1) and is regular in that product; otherwise, we say that $R$ is irregular in $C(U)$ or $C^{\prime \prime}(U)$. A regular involution $R$ of $\mathfrak{U} \circ \mathfrak{B}$ may be written in the form $R=S \circ T$ where $S$ and $T$ are involutions in $\mathfrak{U}$ and $\mathfrak{B}$, respectively. If $U$ is the element of order 2 in $Z(\mathfrak{U} \circ \mathfrak{B})$, we also may have $R=S U \circ T U$. In the case that $\mathfrak{U} \circ \mathfrak{B}$ is a maximal fused product of quasilinear groups, there is only one nontrivial 
involution in $Z(\mathfrak{U} \circ \mathfrak{B})=Z(\mathfrak{U})$. Thus these are the only ways of writing $R$ as the product of two involutions in $\mathfrak{U}$ and $\mathfrak{B}$.

6. Decompositions of centralizers. Let $U$ be a p-involution in $\&$ whose upper centralizer has the decomposition

$$
C^{\prime \prime}(U)=\mathfrak{B} \times_{\mathfrak{a}}\left(\mathfrak{u}_{1} \circ \mathfrak{u}_{2}\right)
$$

as explained in Axiom A. Let $V$ be an involution in $C(U)$. Then $V \in C^{\prime \prime}$. We set

$$
C^{\prime \prime}(U, V)=C_{C^{\prime \prime}(U)}(V)
$$

and call this group the upper centralizer of $U$ and $V$.

If $V$ is a regular involution in $C(U)$, then $V=V_{1} \circ V_{2}$ where $V_{i}$ is an involution in $\mathfrak{u}_{i}, i=1,2$. In this case, $V$ is in the lower centralizer $C^{\prime}(U)$ of $U$. We define the lower centralizer $C^{\prime}(U, V)$ of $U$ and $V$ to be the group

$$
C^{\prime}(U, V)=T\left(C_{T\left(\mathfrak{M}_{1}\right)}\left(V_{1}\right)\right) \circ T\left(C_{T\left(\mathfrak{M}_{2}\right)}\left(V_{2}\right)\right) \text {. }
$$

Obviously this group is determined independently of the representation of $V$ as a product of involutions in $\mathfrak{U}_{1}$ and $\mathfrak{u}_{2}$. We note that because of Condition $\mathrm{A}\left({ }^{(8)}\right.$

$$
C^{\prime}(U, V)=\left(\mathfrak{u}_{1}^{\prime} \times \mathfrak{u}_{1}^{\prime \prime}\right) \circ\left(\mathfrak{u}_{2}^{\prime} \times \mathfrak{u}_{2}^{\prime \prime}\right)
$$

where $\mathfrak{u}_{i}^{\prime}$ and $\mathfrak{u}_{i}^{\prime \prime}$ are involutory quasilinear groups such that rk $\mathfrak{u}_{i}^{\prime}+$ rk $\mathfrak{u}^{\prime}$ $=\mathrm{rk} \mathfrak{U}_{i}, i=1,2$.

If $\mathfrak{S}$ is a group, we designate by $E(\mathfrak{W})$ the maximal perfect subgroup of $\mathfrak{W}$. This is the minimal normal subgroup $\mathfrak{N}$ of $\mathfrak{W}$ such that $\mathfrak{W} / \mathfrak{N}$ is solvable. This group will play an important role in the study of upper and lower centralizers.

Proposition 6.1. Let $U$ be an involution of the first kind and let $V$ be an involution which is regular in $C^{\prime \prime}(U)$. Then $E\left(C^{\prime \prime}(U, V)\right)=E\left(C^{\prime}(U, V)\right)$ $=E(C(U, V))$. If $C^{\prime}(U, V)$ has the decomposition (6.4),

(6.5) $P(E(C(U, V)))=P\left(D\left(\mathfrak{u}_{\mathfrak{1}}^{\prime}\right)\right) \times P\left(D\left(\mathfrak{u}_{1}^{\prime \prime}\right)\right) \times P\left(D\left(\mathfrak{U}_{\mathfrak{z}}^{\prime}\right)\right) \times P\left(D\left(\mathfrak{u}_{\mathfrak{z}}{ }^{\prime}\right)\right)$.

The groups $P\left(D\left(\mathfrak{U}_{i}^{\prime}\right)\right)$ and $P\left(D\left(\mathfrak{U}_{i}^{\prime \prime}\right)\right)$ are isomorphic to $P S L\left(p_{i}^{\prime}, K\right)$ and $\operatorname{PSL}\left(p_{i}^{\prime \prime}, K\right)$, respectively, where $p_{i}^{\prime}=\mathrm{rk} \mathfrak{U}_{i}^{\prime}$ and $p_{i}^{\prime \prime}=\mathrm{rk} \mathfrak{u}_{i}^{\prime \prime}$.

Proof. Since $\mathfrak{U}_{1} \circ \mathfrak{U}_{2}$ is a subgroup of index at most 2 in $C^{\prime \prime}(U), E\left(C^{\prime \prime}(U)\right)$ $=E\left(\mathfrak{U}_{1} \circ \mathfrak{U}_{2}\right)$. This means that $E\left(C^{\prime \prime}(U, V)\right) \subseteq \mathfrak{U}_{1} \circ \mathfrak{U}_{2}$. Let $R \in C(V) \cap\left(\mathfrak{U}_{1} \circ \mathfrak{U}_{2}\right)$. Then $R V_{1} R^{-1} \circ R V_{2} R^{-1}=V_{1} \circ V_{2}$ and $R V_{1} R^{-1}=V_{1} Z$ and $R V_{2} R^{-1}=V_{2} Z^{-1}$ where $Z \in Z\left(\mathfrak{U}_{1} \circ \mathfrak{U}_{2}\right)$. The mapping of $R$ onto the element $Z$ determines a homomorphism of $C(V) \cap\left(\mathfrak{U}_{1} \circ \mathfrak{u}_{2}\right)$ into $Z\left(\mathfrak{u}_{1} \circ \mathfrak{U}_{2}\right)$ whose kernel is the subgroup $C_{\mathfrak{u}_{1}}\left(V_{1}\right) \circ C_{\mathfrak{u}_{2}}\left(V_{2}\right)$. Thus $E\left(C^{\prime \prime}(U, V)\right) \subseteq C_{\mathfrak{u}_{1}}\left(V_{1}\right) \circ C_{\mathfrak{u}_{2}}\left(V_{2}\right)$.

(8) Conditions A, B, C, D, and E refer to the definition of a quasilinear group in [4]. Note that the involutory subgroup of a quasilinear group is quasilinear by Proposition 13.3 of [4]. 
Since $\mathfrak{U}_{i} / T\left(\mathfrak{U}_{i}\right)$ is abelian by Condition $\mathrm{C}$, we have that $E\left(C^{\prime \prime}(U, V)\right)$ $\subseteq C^{\prime}(U, V)$. Since $C^{\prime}(U, V) \subseteq C^{\prime}(U) \subseteq C(U), C^{\prime}(U, V) \subseteq C(U, V)$. Therefore, $E\left(C^{\prime \prime}(U, V)\right)=E\left(C^{\prime}(U, V)\right)=E(C(U, V))$. Furthermore, from (6.4) $E(C(U, V))=E\left(\mathfrak{u}_{1}^{\prime} \times \mathfrak{u}_{1}^{\prime \prime}\right) \circ E\left(\mathfrak{u}_{2}^{\prime} \times \mathfrak{u}_{2}^{\prime \prime}\right)=\left(E\left(\mathfrak{u}_{1}^{\prime}\right) \times E\left(\mathfrak{u}_{1}^{\prime \prime}\right)\right) \circ\left(E\left(\mathfrak{u}_{2}^{\prime}\right) \times E\left(\mathfrak{u}_{2}^{\prime \prime}\right)\right.$. Because of Condition $\mathrm{B}, E\left(\mathfrak{u}_{i}^{\prime}\right)=D\left(\mathfrak{u}_{i}^{\prime}\right)$ and $E\left(\mathfrak{u}_{i}^{\prime \prime}\right)=D\left(\mathfrak{u}_{i}^{\prime \prime}\right)$. In forming the factor group $P(E(C(U, V)))$, the images of the factors $D\left(\mathfrak{u}_{1}^{\prime}\right) \times D\left(\mathfrak{u}_{1}^{\prime \prime}\right)$ and $D\left(\mathfrak{U}_{2}^{\prime}\right) \times D\left(\mathfrak{U}_{2}^{\prime \prime}\right)$ have a nontrivial intersection. Thus it may be seen that $P(E(C(U, V)))=P\left(D\left(\mathfrak{u}_{1}^{\prime}\right) \times D\left(\mathfrak{u}_{1}^{\prime \prime}\right) \times P\left(D\left(\mathfrak{u}_{2}^{\prime}\right) \times D\left(\mathfrak{U}_{2}^{\prime \prime}\right)\right)\right.$. From this (6.5) follows.

By virtue of Principal Theorem $I$, the groups $P\left(D\left(\mathfrak{u}_{i}^{\prime}\right)\right)$ and $P\left(D\left(\mathfrak{U}_{i}^{\prime \prime}\right)\right)$ are isomorphic to $P S L\left(p_{i}^{\prime}, K\right)$ and $P S L\left(p_{i}^{\prime \prime}, K\right)$, respectively.

Proposition 6.2. Let $U$ be an $n / 2$-involution and let $V$ be an involution of (S) which is in $C(U)$ but not in $\mathfrak{U}_{1} \circ \mathfrak{U}_{2}$ of (6.1). Then $E\left(C^{\prime \prime}(U, V)\right)=E(C(U, V))$. The group $P(E(C(U, V)))$ is isomorphic to $P S L(n / 2, K)$.

Proof. Let $W$ be the involution in $\mathscr{W}$ in (6.1). As $V$ is irregular in $C(U)$, it is not in $\mathfrak{U}_{1} \circ \mathfrak{U}_{2}$. Then $V W \in \mathfrak{U}_{1} \circ \mathfrak{U}_{2}$. Naturally the inner automorphism of $\mathfrak{U}_{1} \circ \mathfrak{U}_{2}$ induced by $V W$ leaves $\mathfrak{U}_{1}$ and $\mathfrak{U}_{2}$ invariant. Thus the automorphism of $\mathfrak{U}_{1} \circ \mathfrak{U}_{2}$ induced by $V$ will exchange $\mathfrak{U}_{1}$ and $\mathfrak{U}_{2}$ just as the automorphism induced by $W$. In other words, $V$ satisfies the same conditions which characterize $W$. Thus we may assume that $V \in \mathfrak{W}$.

Just as in the situation of Proposition 6.1, $E\left(C^{\prime \prime}(U, V)\right) \subseteq \mathfrak{U}_{1} \circ \mathfrak{U}_{2}$. We must describe the centralizer of $V$ in $\mathfrak{U}_{1} \circ \mathfrak{U}_{2}$. To do this, let $\mathfrak{U}$ be the subgroup of $\mathfrak{U}_{1} \circ \mathfrak{U}_{2}$ consisting of the elements of the form $R=R_{1} \circ V R_{1} V^{-1}$; clearly $\mathfrak{u} \subseteq C_{\mathfrak{u}_{1}} \circ \mathfrak{u}_{2}(V)$. If $R=R_{1} \circ R_{2}$ is an arbitrary element of $C_{\mathfrak{u}_{1}} \circ \mathfrak{u}_{2}(V)$, then

$$
R=V R_{2} V^{-1} \circ V R_{1} V^{-1}
$$

and $V R_{1} V^{-1}=R_{2} Z$ and $V R_{2} V^{-1}=R_{1} Z^{-1}$ where $Z \in Z\left(\mathfrak{U}_{1} \circ \mathfrak{U}_{2}\right)$. Since the mapping of $R$ onto $Z$ determines a homomorphism of $C \mathfrak{u}_{1} \circ \mathfrak{u}_{2}(V)$ into $Z\left(\mathfrak{U}_{1} \circ \mathfrak{U}_{2}\right)$ with kernel $\mathfrak{u}, E\left(C^{\prime \prime}(U, V)\right) \subseteq \mathfrak{u}$. Hence $E\left(C^{\prime \prime}(U, V)\right) \subseteq E(\mathfrak{u})$.

Clearly $\mathfrak{U}$ is isomorphic to $\mathfrak{U}_{1}$, which is a quasilinear group of rank $n / 2$. Hence $E(\mathfrak{U})=D(\mathfrak{U})$ and $P(E(\mathfrak{U}))=P(D(\mathfrak{U}))$. By Principal Theorem I, $P(D(\mathfrak{U}))$ is isomorphic to $P S L(n / 2, K)$. Because $E(\mathfrak{u}) \subseteq D\left(\mathfrak{U}_{1}\right) \circ D\left(\mathfrak{U}_{2}\right)$ $\subseteq C^{\prime}(U) \subseteq C(U), E(\mathfrak{u})$ is contained in $E(C(U, V))$. But $E(C(U, V))$ $\subseteq E\left(C^{\prime \prime}(U, V)\right) \subseteq E(\mathfrak{U})$. Hence $E(\mathfrak{U})$ is the maximal perfect subgroup of $\bar{C}(U, V)$ as well as of $C^{\prime \prime}(U, V)$. This shows that $E\left(C^{\prime \prime}(U, V)\right)=E(C(U, V))$ $=E(\mathfrak{U})$ and proves the proposition.

Proposition 6.3. Let $U$ be an involution of the first kind and let $V$ be an involution in $\mathfrak{U}_{1} \circ \mathfrak{U}_{2}$ of (6.1) which is an irregular involution in $C^{\prime \prime}(U)$. Then $E\left(C^{\prime \prime}(U, V)\right)=E(C(U, V))$. Furthermore $p=\mathrm{rk} U_{1}$ and $n-p=\mathrm{rk} U_{2}$ are even and $P(E(C(U, V)))$ is the direct product of groups isomorphic to $P S L(p / 2, L)$ and $P S L((n-p) / 2, L)$, respectively, where $L$ is a quadratic extension of $K$.

Proof. In this case $V$ is an element of $\mathfrak{l}_{1} \circ \mathfrak{U}_{2}$ with a representation 
$V=V_{1} \circ V_{2}$ where $V_{1}^{2}=V_{2}^{-2}=A$ is a nonsquare in the center $Z\left(\mathfrak{U}_{1}\right)=Z\left(\mathfrak{U}_{2}\right)$ of $\mathfrak{U}_{1} \circ \mathfrak{U}_{2}$. The argument given in the proof of Proposition 6.1 now yields that $E\left(C^{\prime \prime}(U, V)\right) \subseteq C_{\mathfrak{u}_{1}}\left(V_{1}\right) \circ C_{\mathfrak{u}_{2}}\left(V_{2}\right)$. Thus we must investigate the centralizers $C_{\mathfrak{u}_{i}}\left(V_{i}\right), i=1,2$.

We will make use of the epimorphisms $\Phi: \mathfrak{U}_{i} \rightarrow P\left(\mathfrak{U}_{i}\right)$ and $\Lambda: G L\left(p_{i}, K\right)$ $\rightarrow P\left(\mathfrak{U}_{i}\right)$ described $\left({ }^{9}\right)$ in Propositions I.6.1 and I.9.1. It is also convenient to identify $P\left(\mathfrak{U}_{i}\right)$ with $P G L\left(p_{i}, K\right)$. We claim that the involution $\Phi V_{i}$ is an involution of the second kind in $P G L\left(p_{i}, K\right)$. For, if this is not the case, there exists an involution $X$ of the first kind in $\mathfrak{U}_{i}$ such that $\Phi X=\Phi V_{i}$ by virtue of Proposition I.9.1. Then $\Phi\left(X V_{i}\right)=1$; hence $V_{1}=X Z$ where $Z \in Z\left(\mathfrak{U}_{1}\right)$. This implies that $V_{1}^{2}=A=Z^{2}$, which is a contradiction.

Because $\Phi \mathfrak{U}_{1}=\Lambda G L\left(p_{i}, K\right)$, there exists an element $\bar{V}_{i} \in G L\left(p_{i}, K\right)$ such that $\Phi V_{i}=\Lambda \bar{V}_{i}$. Then $\bar{V}_{i}$ is a projective involution of the second kind in $G L\left(p_{i}, K\right)$ as $\Lambda \bar{V}_{i}$ is an involution of the second kind in $P G L\left(p_{i}, K\right)$. As we mentioned in $\S 3$, the centralizer of $\bar{V}_{i}$ is isomorphic to $G L\left(p_{i} / 2, L\right)$ where $L$ is a quadratic extension of $K$. Thus $E\left(C_{G L\left(p_{i}, K\right)}\left(\bar{V}_{i}\right)\right)=S L\left(p_{i} / 2, L\right)$.

Now both $\Lambda C_{G L\left(p_{i}, K\right)}\left(\bar{V}_{i}\right)$ and $\Phi C_{\mathfrak{u}_{i}}\left(V_{i}\right)$ are subgroups of $C_{\Lambda G L\left(p_{i}, K\right)}\left(\Lambda \bar{V}_{i}\right)$ $=C_{\Phi \mathfrak{u}_{i}}\left(\Phi V_{i}\right)$ of index at most 2. Therefore, $E\left(C_{\Phi \mathfrak{u}_{i}}\left(\Phi V_{i}\right)\right)=E\left(\Lambda C_{G L\left(p_{i}, K\right)}\left(\bar{V}_{i}\right)\right)$ and $E\left(C_{\Phi \mathfrak{u}_{i}}\left(\Phi V_{i}\right)\right)=E\left(\Phi C_{\mathfrak{u}_{i}}\left(V_{i}\right)\right)$. Since the homomorphic image of a maximal perfect normal subgroup is a perfect normal subgroup of the image group whose factor group is solvable, the image of a maximal perfect subgroup is a maximal perfect subgroup of the image group. Thus $\Phi E\left(C_{\mathfrak{u}_{i}}\left(V_{i}\right)\right)$ $=E\left(\Phi C_{\Phi \mathfrak{u}_{i}}\left(\Phi V_{i}\right)\right)=E\left(\Lambda C_{G L\left(p_{i}, K\right)}\left(\Lambda \bar{V}_{i}\right)\right)=E\left(C_{G L\left(p_{i}, K\right)}\left(\bar{V}_{i}\right)\right)$.

Since the kernels of $\Phi$ and $\Lambda$ are the centers of $\mathfrak{u}_{i}$ and $G L\left(p_{i}, K\right)$, respectively, it follows that $P\left(E\left(C_{\mathfrak{u}_{i}}\left(V_{i}\right)\right)\right)$ and $P\left(E\left(\Phi C_{\mathfrak{u}_{i}}\left(V_{i}\right)\right)\right)$ as well as $P\left(E\left(C_{a L\left(p_{i}, K\right)}\left(\bar{V}_{i}\right)\right)\right)$ and $P\left(E\left(\Lambda C_{G L\left(p_{i}, K\right)}\left(\bar{V}_{i}\right)\right)\right)$ are isomorphic. Thus $P\left(E\left(C_{\mathfrak{u}_{i}}\left(V_{i}\right)\right)\right)$ is isomorphic to $P S L\left(p_{i} / 2, L\right)$.

As $E\left(C^{\prime \prime}(U, \quad V)\right)=E\left(C_{\mathfrak{u}_{1}}\left(V_{1}\right) \circ C_{\mathfrak{u}_{2}}\left(V_{2}\right)\right)=E\left(C_{\mathfrak{u}_{1}}\left(V_{1}\right)\right) \circ E\left(C_{\mathfrak{u}_{2}}\left(V_{2}\right)\right)$, $P\left(E\left(C^{\prime \prime}(U, V)\right)\right)=P\left(E\left(C_{\mathfrak{u}_{1}}\left(V_{1}\right)\right)\right) \times P\left(E\left(C_{\mathfrak{u}_{2}}\left(V_{2}\right)\right)\right)$, and this group is isomorphic to $P S L(p / 2, L) \times P S L((n-p) / 2, L)$. Also note that $E\left(C_{\mathfrak{u}_{i}}\left(V_{i}\right)\right)$ $\subseteq D\left(\mathfrak{U}_{i}\right) \subseteq T\left(\mathfrak{U}_{i}\right)$. Therefore, $E\left(C^{\prime \prime}(U, V)\right) \subseteq C^{\prime}(U)$. This implies that $E\left(C^{\prime \prime}(U, V)\right)=E(C(U, V))$ as before. Hence the proposition is proved.

The direct factors $P S L(p, K)$ or $P S L(p, L)$ which appear in a decomposition of $P(E(C(U, V)))$ will be called the normal factors of $C(U, V)$. When $p=1$, they are identity groups. The integer $p$ will be called the rank of the corresponding normal factor. A group $\operatorname{PSL}(p, K)$ or $\operatorname{PSL}(p, L)$ of rank $p>1$ is either simple or the alternating group on 4 letters when $K$ and $L$ have characteristic not 2 by virtue of the Dickson-Dieudonné Theorem [2, p. 38]. Thus they are indecomposable and uniquely determined up to isomorphism as factors of $P(E(C(U, V)))$ by the Krull-Schmidt Theorem. We will assume that

( ${ }^{9}$ Actually these propositions treat the case that $\mathfrak{U}_{i}$ is an involutory quasilinear group, not a full quasilinear group as here. Nevertheless these results except for the last statement of Proposition 1.9.1 hold in this case by the arguments given in [4]. 
there are as many normal factors of rank 1 in the decomposition of $P(E(C(U, V)))$ in the situation of Proposition 6.1 as necessary for the sum of the ranks of the normal factors to be $n$. By a theorem of Dieudonne [1, p. 22] the normal factors of rank greater than 1 are determined up to isomorphism by specifying the field $K$ or $L$ and their ranks. This fact is of critical importance in the next section.

\section{Centralizers of involutions.}

Proposition 7.1. Let $U$ be a 1-involution of (S) and suppose that $V$ is an involution of (S) belonging to $C(U)$. Then $V$ is an involution of the first kind in 8 ; $V$ is a regular involution in $C(U)$ and $U$ is a regular involution in $C(V)$.

Proof. By Axiom A, $V$ is of the first kind in $\$$.

Because $U$ is a 1 -involution, $C^{\prime \prime}(U)$ has the decomposition

$$
C^{\prime \prime}(U)=\mathfrak{u}_{1} \circ \mathfrak{u}_{2}
$$

as the maximal fused product of full quasilinear groups of ranks $n-1$ and 1 , respectively. Since a quasilinear group of rank 1 is commutative, it follows that $\mathfrak{U}_{2}=Z\left(\mathfrak{U}_{2}\right)=Z\left(\mathfrak{U}_{1}\right) \subseteq \mathfrak{U}_{1}$. Therefore, (7.1) simplifies to

$$
C^{\prime \prime}(U)=\mathfrak{u}_{1} \text {. }
$$

Then $V$ must be an involution in $\mathfrak{U}_{1}$; consequently, $V$ is a regular involution in $C(U)$. Thus by the definition of a full quasilinear group,

$$
C^{\prime \prime}(U, V)=\left(\mathfrak{u}_{1}^{\prime} \times \mathfrak{u}_{\mathfrak{1}}^{\prime \prime}\right) \circ \mathfrak{U}_{2}=\mathfrak{u}_{1}^{\prime} \times \mathfrak{u}_{1}^{\prime \prime}
$$

where $\mathfrak{U}_{1}^{\prime}$ and $\mathfrak{u}_{1}^{\prime \prime}$ are full quasilinear groups of ranks $p$ and $n-p-1$, respectively. Thus the normal factors of $C(U, V)$ have ranks $p, n-p-1$, and 1 by Proposition 6.1.

Because $V$ is an involution of the first kind in $\mathcal{S}$, we have the decomposition

$$
C^{\prime \prime}(V)=\mathfrak{B} \times_{\mathfrak{B}}\left(\mathfrak{B}_{1} \circ \mathfrak{B}_{2}\right)
$$

determined in accordance with Axiom A. We form $C^{\prime \prime}(V, U)$ from (7.4) to obtain normal factors of it and $C(V, U)=C(U, V)$. These normal factors must be those of $C(U, V)$ determined from (7.3). Because of this, it follows from Proposition 6.3 that $U$ is not an irregular involution in $C(V)$ unless $V$ is an $n / 2$-involution and $U$ is not in $\mathfrak{B}_{1} \circ \mathfrak{B}_{2}$. In this case, it follows from Proposition 6.2 that the only normal factor of rank greater than 1 is $P S L(n / 2, K)$. Thus only one of $p$ and $n-p-1$ is greater than 1 . Hence we may take $p=n / 2$ and $n-p-1=1$. This yields that $n=4$.

To eliminate this case, we choose three mutually commuting 1 -involutions, $T_{1}, T_{2}$, and $T_{3}$, of the quasilinear group $\left({ }^{10}\right) \mathfrak{U}_{1}$ in the decomposition groups $\mathfrak{u}_{1}^{\prime}$ and $\mathfrak{u}_{1}^{\prime \prime}$. We may suppose that $T_{1}$ and $T_{2}$ belong to one of these

(10) These are the involutions in the class $\Omega_{1}\left(\mathfrak{U}_{1}\right)$ (cf. [4]). 
groups, say $\mathfrak{U}_{1}^{\prime}$. Then $\mathfrak{U}_{\mathfrak{1}}^{\prime \prime}$ has rank 1 and $T_{\mathfrak{z}}$ is the only nontrivial involution in $\mathfrak{U}_{1}^{\prime \prime}$. As $\mathfrak{U}_{1}^{\prime \prime}$ is isomorphic to $G L(2, K)$ by definition (cf. $\left[4\right.$, p. 483]), $T_{1}$ and $T_{2}$ are not only conjugate in $\mathfrak{U}_{1}$ but also in $D\left(\mathfrak{u}_{1}^{\prime}\right)$. Since $D\left(\mathfrak{U}_{1}^{\prime}\right) \subseteq T\left(\mathfrak{u}_{1}^{\prime}\right)$ $\subseteq C^{\prime}(U, V) \subseteq C(U, V), T_{1}$ and $T_{2}$ are conjugate in $C(U, V)$. Then also $T_{1} T_{2}$ and $T_{2} T_{3}$ are conjugate in $C(U, V)$. These are the only instances of conjugacy among the eight elements in the group $\mathfrak{T}$ generated by $T_{1}, T_{2}$, and $T_{2}$.

Among the involutions in $\mathfrak{I}$ is $U$, which is not in $\mathfrak{B}_{1} \circ \mathfrak{B}_{2}$. Since $\left[C^{\prime \prime}(V): \mathfrak{B}_{1} \circ \mathfrak{B}_{2}\right]=2$, four of the involutions of $\mathfrak{T}$, say $1, S_{1}, S_{2}$, and $S_{1} S_{2}$, are in $\mathfrak{B}_{1} \circ \mathfrak{B}_{2}$. The other involutions, which are not in $\mathfrak{B}_{1} \circ \mathfrak{B}_{2}$; will be $U$, $U S_{1}, U S_{2}$, and $U S_{1} S_{2}$. One of the pairs of involutions in $\mathfrak{T}$ which are conjugate in $C(U, V)$ must belong to $\mathfrak{B}_{1} \circ \mathfrak{B}_{2}$ as neither pair contains $U$. We assume that this pair is $S_{1}$ and $S_{2}$. Then $U S_{1}$ and $U S_{2}$ is the other conjugate pair. Then either $S_{1}=T_{i}$ and $S_{2}=T_{j}, i \neq j$ and $i, j=1,2$, or $S_{1}=T_{i} T_{3}$ and $S_{2}=T_{j} T_{3}$. Furthermore, $U S_{1}$ and $U S_{2}$ are also one of the pairs $T_{1}$ and $T_{2}$ or $T_{1} T_{3}$ and $T_{2} T_{3}$. In any event, $S_{1} S_{2}=T_{1} T_{2}$, which is in the center of $U_{1}^{\prime}$ but not in the center of $C(U)$. Thus $S_{1} S_{2}$ is either $V$ or $U V$. Since $S_{1} S_{2} \in V_{1} \circ V_{2}, S_{1} S_{2}=V$. As $S_{2}$ is conjugate to $S_{1}$ in $C(U, V), V \in D(C(U, V))$.

Next observe from Proposition 6.1 and (7.3) that $E(C(U, V))=D\left(\mathfrak{u}_{1}^{\prime}\right)$ $\times D\left(\mathfrak{U}_{1}^{\prime \prime}\right)$ since $D\left(\mathfrak{U}_{1}^{\prime}\right)$ and $D\left(\mathfrak{U}_{1}^{\prime \prime}\right)$ are perfect normal subgroups of $\mathfrak{U}_{1}^{\prime}$ and $\mathfrak{U}_{\mathfrak{i}}{ }^{\prime}$, respectively. Thus it follows that $E(C(U, V))=D(C(U, V))$. Hence $V \in E(C(U, V))$.

In the argument of Proposition 6.2, we identified $E(C(U, V))$. In the present notation, it may be described as the subgroup of $\mathfrak{B}_{1} \circ \mathfrak{B}_{2}$ consisting of elements of the form $R \circ U R U^{-1}$. To establish a contradiction, we will show that $E(C(U, V))$ does not contain $V$. Indeed, if it did, then $V=1 \circ V$ $=R \circ U R U^{-1}$. This is possible only if $R \in Z\left(\mathfrak{B}_{1} \circ \mathfrak{B}_{2}\right)=Z\left(\mathfrak{B}_{1}\right)$. Since $p=n / 2$ $=2, \mathfrak{B}_{1}$ is isomorphic to $G L(2, K)$ and $D\left(\mathfrak{B}_{1}\right)$ is isomorphic to $S L(2, K)$ (cf. [4, p. 483]). The center of $S L(2, K)$ is of order 2. Thus $Z\left(D\left(\mathfrak{B}_{1}\right)\right)$ is generated by $V$. This means that $R=1$ or $R=V$. In either case, $V=R U R U^{-1}$ leads to $V=1$, which is a contradiction. Thus $V \in E(C(U, V))$, which is our promised contradiction. This proves the proposition.

Proposition 7.2. Let $U$ be an $n / 2$-involution and let $V$ be a 1-involution in $C(U)$. If $C^{\prime \prime}(U)$ has the decomposition (6.1), then the involutions of $C^{\prime \prime}(U)$ which commute with $V$ belong to $\mathfrak{U}_{1} \circ \mathfrak{U}_{2}$.

Proof. Proposition 7.1 implies that $V \in \mathfrak{U}_{1} \circ \mathfrak{U}_{2}$ and that $V$ has the representation $V=V_{1} \circ V_{2}$, where $V_{1}$ and $V_{2}$ are involutions. Then $C^{\prime}(U, V)$ has the decomposition (6.4). We argued in the proof of Proposition 6.2 that any involution $X$ in $C^{\prime \prime}(U)$ but not in $\mathfrak{U}_{1} \circ \mathfrak{U}_{2}$ satisfies the same conditions as does the involution $W$ in the group $W$ of (6.1). So we may reduce our considerations to discussing the possibility that $W$ commutes with the involutions of $C(U, V)$. If this is the case, then $W V W^{-1}=V$. Thus, as $W \mathfrak{U}_{1} W^{-1}=\mathfrak{U}_{2}$ and $W \mathfrak{U}_{2} W^{-1}=\mathfrak{l}_{1}, W V_{1} W^{-1}=V_{2} Z$ and $W V_{2} W^{-1}=V_{1} Z^{-1}$ with $Z \in Z\left(\mathfrak{U}_{1} \circ \mathfrak{u}_{2}\right)$. 
From this it follows that if $R \in C_{\mathfrak{u}_{1}}\left(V_{1}\right)$, then $W R W^{-1} \in C_{\mathfrak{u}_{2}}\left(V_{2}\right)$ and vice versa. Thus we may choose $\mathfrak{u}_{2}^{\prime}=W \mathfrak{u}_{1}^{\prime} W^{-1}$ and $\mathfrak{U}_{1}^{\prime \prime}=W \mathfrak{u}_{2}^{\prime \prime} W^{-1}$. This means that the normal factors of a given rank that belong to $C(U, V)$ occur in pairs.

Since $V$ is a 1-involution, it follows that $C^{\prime \prime}(U, V)$ has a decomposition similar to (7.3). In particular, $C(U, V)$ has but three normal factors of ranks $p, n-p-1$, and 1 . This yields a contradiction. Hence involutions such as $W$ do not belong to $C(U, V)$, and the proposition is proved.

We now define two commuting involutions $U$ and $V$ of the first kind in (S) to be compatible if there exists a 1-involution in $C(U, V)$.

Proposition 7.3. Let $U$ and $V$ be commuting compatible involutions of the first kind in 8 . Then $V$ is regular in $C^{\prime \prime}(U)$ and $U$ is regular in $C^{\prime \prime}(V)$.

Proof. We need only prove the first statement. We will obtain a contradiction of the assumption that $V$ is irregular in $C^{\prime \prime}(U)$. Let $W$ be a 1 -involution in $C(U, V)$. Let $C^{\prime \prime}(U)$ have the decomposition (6.1). Proposition 7.1 implies that $W \in \mathfrak{U}_{1} \circ \mathfrak{U}_{2}$ and that relative to this decomposition $W=W_{1} \circ W_{2}$ where $W_{1}$ and $W_{2}$ are involutions. Proposition 7.2 implies that $V \in \mathfrak{U}_{1} \circ \mathfrak{U}_{2}$. Let $p=\mathrm{rk} \mathfrak{U}_{1}$; then $\mathrm{rk} \mathfrak{U}_{2}=n-p$.

As $W$ is a 1 -involution, $U$ is a regular involution in $C^{\prime \prime}(W)$. Then we have seen that $C(W, U)$ has but three normal factors of ranks $p, n-p-1$, and 1 . At least one and at most two of these normal factors is nontrivial; when there is only one nontrivial normal factor, it has rank $n-2$.

Because $W$ is regular in $C(U)$, Proposition 6.1 implies that there is a decomposition of $C^{\prime}(U, W)$ from which the normal factors of $C(U, W)$ may be obtained. Since there are only three of these,

$$
C^{\prime}(U, W)=\left(\mathfrak{U}_{1}^{\prime} \times \mathfrak{u}_{1}^{\prime \prime}\right) \circ T\left(\mathfrak{U}_{2}\right)
$$

where $\mathfrak{U}_{1}^{\prime}$ and $\mathfrak{u}_{1}^{\prime \prime}$ are involutory quasilinear groups and $T\left(\mathfrak{U}_{2}\right)$ is the involutory quasilinear subgroup of the factor $\mathfrak{H}_{2}$. This means that $T\left(C_{T\left(\mathfrak{l}_{2}\right)}\left(W_{2}\right)\right)$ $=T\left(\mathfrak{U}_{2}\right)$. Hence $W_{2} \in Z\left(T\left(\mathfrak{U}_{2}\right)\right) \subseteq Z\left(\mathfrak{U}_{2}\right)$ because of Condition D. Let $V=V_{1} \circ V_{2}$ be the representation of $V$ in $\mathfrak{u}_{1} \circ \mathfrak{U}_{2}$. Since $V W=W V, V_{1} W_{1} V_{1}^{-1}=W_{1} Z$ and $V_{2} W_{2} V_{2}^{-1}=W_{2} Z^{-1}$ where $Z \in Z\left(\mathfrak{U}_{1} \circ \mathfrak{U}_{2}\right)$. As $W_{2} \in Z\left(\mathfrak{U}_{2}\right), Z=1$.

As $V$ is an irregular involution in $C(U), V_{1}^{2}=V_{2}^{-2}=A$ where $A$ is a nonsquare in $Z\left(\mathfrak{U}_{1} \circ \mathfrak{U}_{2}\right)=Z\left(\mathfrak{U}_{1}\right)=Z\left(\mathfrak{U}_{2}\right)$. Then compare $\mathfrak{U}_{1}$ with $G L(p, K)$ by the homomorphisms $\Phi: U_{1} \rightarrow P\left(\mathfrak{U}_{1}\right)$ and $\Lambda: G L(p, K) \rightarrow P G L(p, K)$ as we did in the proof of Proposition 6.2. There we saw that $\Phi V_{1}$ is an involution of the second kind in $P\left(\mathfrak{U}_{1}\right)=P G L(p, K)$ and that $\Phi W_{1}$ is an involution of the first kind in $P G L(p, K)$. This means that $\Phi W_{1}$ is in the class $\mathfrak{R}_{r}\left(P\left(\mathfrak{U}_{1}\right)\right)$ where $r$ and $p-r$ are even. Then by Proposition I.9.1, $W_{1}$ is an $r$-involution of the quasi-linear groups $\mathfrak{u}_{1}$. Thus rk $\mathfrak{u}_{\mathfrak{i}}^{\prime}=r$ and $\mathrm{rk} \mathfrak{u}_{\mathfrak{1}}^{\prime \prime}=p-r$ are even.

Because one of the decomposition groups in (7.5) has rank $1, \mathrm{rk} \mathfrak{U}_{2}=1$. Thus $\mathfrak{U}_{2}=Z\left(\mathfrak{U}_{2}\right)$. Then in $Z\left(\mathfrak{U}_{2}\right), V_{2}^{2}=A^{-1}$. This contradicts the fact that $A$ is a nonsquare in $Z\left(\mathfrak{U}_{2}\right)=Z\left(\mathfrak{U}_{1} \circ \mathfrak{U}_{2}\right)$. The proposition is proved. 
Proposition 7.4. Let $U$ be an involution of the first kind which commutes with a 1-involution in $\left(5\right.$. Then the lower centralizer $C^{\prime}(U)$ is the subgroup of $C(U)$ which is generated by the involutions of the first kind in $\mathbb{S}$ which commute with and are compatible with $U$. These involutions are regular in $C(U)$.

Proof. First, by Proposition 7.3, all the involutions of the first kind which commute with and are compatible with $U$ are regular in $C(U)$. Thus they generate a subgroup of $C^{\prime}(U)$.

On the other hand, $U$ commutes with a 1 -involution $X$, which must be regular in $C(U)$ by virtue of Proposition 7.1. Let $X=X_{1} \circ X_{2}$ be the representation of $X$ relative to the decomposition $C^{\prime}(U)=\mathfrak{U}_{1} \circ \mathfrak{U}_{2}$ where $X_{1}$ is a $p_{1}$-involution of the quasilinear group $U_{1}$ and $X_{2}$ is an $p_{2}$-involution of the quasilinear group $\mathfrak{U}_{2}$. Now let $R=R_{1} \circ R_{2}$ be an arbitrary involution in $C^{\prime}(U)$ with $R_{i}$ an involution in $\mathfrak{U}_{i}$. Since $C^{\prime}(U)$ is generated by such involutions as $R$, we must show that they are compatible with $U$ to prove the proposition.

In the quasilinear group $\mathfrak{U}_{i}$, there is a maximal set of mutually commuting involutions containing $R_{i}$ (cf. $[4, \S \S 7,8]$ ). Let $T_{i}$ be a product of $p_{i}$ mutually commuting 1-involutions of $\mathfrak{U}_{i}$ that belong to this set. Then $T_{i}$ is conjugate to $X_{i}$ in $\mathfrak{U}_{i}$ by Theorem I.8.5. Thus $T=T_{1} \circ T_{2}$ is conjugate to $X$ in $C^{\prime}(U)$ and thus is a 1-involution of $\&$ which commutes with $R$. This proves the proposition.

Now we wish to treat sets of mutually commuting involutions. A set of mutually commuting involutions of the first kind is said to be compatible if there is a 1-involution which commutes with all of them.

Proposition 7.5. Let $\left\{U_{1}, U_{2}, \cdots, U_{t}\right\}$ be a compatible mutually commuting set of involutions of the first kind in $\$$. Let

$$
C^{\prime}\left(U_{i}\right)=\mathfrak{U}_{i}^{\prime} \circ \mathfrak{U}_{i}^{\prime \prime}
$$

be a decomposition of $C^{\prime}\left(U_{i}\right)$ into involutory quasilinear groups. Then $U_{j}$ is regular in $C\left(U_{i}\right), j=1,2, \cdots, t$, and has the representation $U_{j}=U_{j}^{\prime} \circ U_{j}^{\prime \prime}$ relative to (7.6) where $U_{j}^{\prime}$ and $U_{j}^{\prime \prime}$ are involutions. Furthermore, the components $U_{j}^{\prime}$ and $U_{k}^{\prime}$ as well as the components $U_{j}^{\prime \prime}$ and $U_{k}^{\prime \prime}, j, k=1,2, \cdots, t$, commute.

Proof. It follows from Proposition 7.3 that all the involutions $U_{j}$ are regular in $C\left(U_{i}\right)$. Consequently, they have the stated representation in $C^{\prime}\left(U_{i}\right)$. Consequently, they have the stated representation in $C^{\prime}\left(U_{i}\right)$. Because $U_{j}$ and $U_{k}$ commute, $U_{j}^{\prime} U_{k}^{\prime}=U_{k}^{\prime} U_{j}^{\prime} Z$ and $U_{j}^{\prime \prime} U_{k}^{\prime \prime}=U_{k}^{\prime \prime} U_{j}^{\prime \prime} Z^{-1}$ where $Z \in Z\left(\mathfrak{U}_{i}^{\prime} \circ \mathfrak{U}_{i}^{\prime \prime}\right)$. We wish to show that $Z=1$.

In $C\left(U_{i}\right)$ there is a 1 -involution $W$ which commutes with all the involutions $U_{1}, U_{2}, \cdots, U_{t}$. Because of Proposition 7.1, $W \in C^{\prime}\left(U_{i}\right)$. Let $W=W^{\prime} \circ W^{\prime \prime}$ be the representation of $W$ relative to (7.6). We argued in the proof of Proposition 7.3 that any involution in $C(U)$ which commutes with 
$W$ has components which commute with $W^{\prime}$ and $W^{\prime \prime}$. Also corresponding to (7.5), we have (after at most exchanging the notation designating $\mathfrak{u} \mathfrak{l}$, and $\left.\mathfrak{u}_{i}^{\prime \prime}\right)$

$$
C^{\prime}\left(U_{i}, W\right)=\left(\mathfrak{B}_{1} \times \mathfrak{W}_{2}\right) \circ \mathfrak{u}_{i}^{\prime \prime}
$$

where one of the decomposition groups $\mathfrak{B}_{1}, \mathfrak{W}_{2}$ or $\mathfrak{u}_{i}^{\prime \prime}$ has rank 1 . Should $\mathfrak{u}_{i}^{\prime \prime}$ have rank 1 , then $\mathfrak{u}_{i}^{\prime \prime}=Z\left(\mathfrak{u}_{i}^{\prime \prime}\right) \supseteq Z\left(\mathfrak{u}_{i}^{\prime} \circ \mathfrak{u}_{i}^{\prime \prime}\right)$. This means that $U_{j}^{\prime \prime}$ and $U_{k}^{\prime \prime}$ commute. Hence $Z=1$ in this case.

Thus there remains the case where, in the involutory quasilinear group $\mathfrak{u}_{i}^{\prime}$, the involutory centralizer $T\left(C_{\mathfrak{u}_{\mathfrak{i}^{\prime}}}\left(W^{\prime}\right)\right)$ has the decomposition

$$
T\left(C_{\mathfrak{u}^{\prime}}\left(W^{\prime}\right)\right)=\mathfrak{B}_{1} \times \mathfrak{B}_{2}
$$

in which one of the decomposition groups, say $\mathfrak{W}_{1}$, has rank 1 . Relative to (7.8), we have the representation $U_{j}^{\prime}=U_{11}^{\prime} \times U_{j 2}^{\prime}$ and $U_{k}^{\prime}=U_{k 1}^{\prime} \times U_{k 2}^{\prime}$. We may apply Proposition I.9.1 and now conclude that (7.8) is a normal decomposition. This means that $Z$ has the representation $Z=Z_{1} \times Z_{2}$ relative to (7.8); and if $Z \neq 1$, neither $Z_{1}=1$ nor $Z_{2}=1$. As $W_{1}$ is commutative, $U_{k 1}^{\prime} U_{k 1}^{\prime}$ $=U_{k 1}^{\prime} U_{j 1}^{\prime}$ and $Z_{1}=1$. Thus $Z=1$, and the proposition is proved.

Let $S=\left\{U_{1}, U_{2}, \cdots, U_{t}\right\}$ be a compatible set of mutually commuting involutions of the first kind. Using the notation of Proposition 7.5, we define the lower centralizer $C^{\prime}(\delta)=C^{\prime}\left(U_{1}, U_{2}, \cdots, U_{t}\right)$ to be the group

$$
C^{\prime}(\delta)=T\left(C_{\mathfrak{u}_{\mathfrak{i}^{\prime}}}\left(U_{1}^{\prime} U_{2}^{\prime}, \cdots, U_{!}^{\prime}\right)\right) \circ T\left(C_{\mathfrak{u}_{\mathfrak{i}^{\prime \prime}}}\left(U_{1}^{\prime \prime}, U_{2}^{\prime}, \cdots, U_{!^{\prime}}^{\prime}\right)\right) \text {. }
$$

Note that by Condition A, each of the groups will be a direct product of involutory quasilinear groups, the sum of whose ranks is the rank of $\mathfrak{u}_{i}^{\prime}$ and of $\mathfrak{u}{ }_{i}^{\prime \prime}$. That the lower centralizer of (7.9) actually does not depend on the involution $U_{i}$ used to define it follows from the following proposition.

Proposition 7.6. Let $\delta=\left\{U_{1}, U_{2}, \cdots, U_{t}\right\}$ be a compatible set of mutually commuting involutions of the first kind. Then $C^{\prime}(\delta)$ is generated by the involutions $X$ such that $U_{1}, U_{2}, \cdots, U_{t}, X$ form a compatible set of mutually commuting involutions of the first kind.

Proof. First let $X$ be an involution satisfying the condition of the proposition. Then by Proposition 7.4, $X \in C^{\prime}\left(\mathfrak{U}_{i}\right)$. Hence relative to the decomposition (7.6) of $C^{\prime}\left(\mathfrak{u}_{i}\right), X=X^{\prime} \circ X^{\prime \prime}$ where $X^{\prime}$ and $X^{\prime \prime}$ are involutions. Proposition 7.5 applied to the set $U_{1}, U_{2}, \cdots, U_{t}, X$ implies that the component $X^{\prime}$ commutes with the components $U_{j}^{\prime}$ and that the component $X^{\prime \prime}$ commutes with the components $U_{j}^{\prime \prime}$. Thus $X \in C^{\prime}(\delta)$.

Conversely, suppose that $X$ is an involution in $C^{\prime}(\delta)$ such that $X=X^{\prime} \circ X^{\prime \prime}$ where $X^{\prime}$ and $X^{\prime \prime}$ are involutions in $\mathfrak{u}_{i}^{\prime}$ and $\mathfrak{u}_{i}^{\prime \prime}$, respectively. Then $C^{\prime}(\mathcal{S})$ is generated by involutions such as $X$. We must show that $X$ satisfies the condition of the proposition.

Because of the form of (7.9), it follows that $X^{\prime}$ commutes with all the 
components $U_{j}^{\prime}$ and $X^{\prime \prime}$ commutes with all the components $U_{j}^{\prime \prime}$. Let $W=W^{\prime} \circ W^{\prime \prime}$ be a 1 -involution in $C^{\prime}\left(\mathfrak{U}_{i}\right)$. By the argument of Proposition 7.4 , there exists an involution whose components in $\mathfrak{u}_{i}^{\prime}$ and in $\mathfrak{u}_{i}^{\prime \prime}$ are conjugate, respectively, to $W^{\prime}$ and $W^{\prime \prime}$ and which commute with the components $\mathfrak{u}_{j}^{\prime}$ and $\mathfrak{u}_{j}^{\prime \prime}$ and with $X^{\prime}$ and $X^{\prime \prime}$ as well. This proves that $U_{1}, U_{2}, \cdots, U_{\imath}$, $X$ form a compatible set of mutually commuting involutions. Thus the proposition is proved.

There may be many ways of describing the lower centralizer of a compatible set of mutually commuting involutions $U_{1}, U_{2}, \ldots, U_{t}$ of the first kind. For example, we may form first the decomposition (7.10) of the lower centralizer

$$
C^{\prime}\left(U_{1}\right)=\mathfrak{l}_{1}^{\prime} \circ \mathfrak{U}_{1}^{\prime \prime}
$$

where $\mathfrak{u}_{1}^{\prime}$ and $\mathfrak{u}_{1}^{\prime \prime}$ are involutory quasilinear groups. Relative to (7.10), $U_{2}=U_{2}^{\prime} \circ U_{2}^{\prime \prime}$ where $U_{2}^{\prime}$ and $U_{2}^{\prime \prime}$ are involutions. Hence we may next form the decomposition

$$
\begin{aligned}
C^{\prime}\left(U_{1}, U_{2}\right) & =T\left(C_{\mathfrak{u}_{1}}\left(U_{2}^{\prime}\right)\right) \circ T\left(C_{\mathfrak{u}_{1}{ }^{\prime}}\left(U_{2}^{\prime \prime}\right)\right) \\
& =\left(\mathfrak{X}^{\prime} \times \mathfrak{X}^{\prime \prime}\right) \circ\left(\mathfrak{Z}^{\prime} \times \mathfrak{Y}^{\prime \prime}\right)
\end{aligned}
$$

where the groups $\mathfrak{X}^{\prime}, \mathfrak{X}^{\prime \prime}, \mathfrak{Y}^{\prime}$, and $\mathfrak{Y}^{\prime \prime}$ are involutory quasilinear groups such that rk $\mathfrak{X}^{\prime}+\mathrm{rk} \mathfrak{X}^{\prime \prime}=\mathrm{rk} \mathfrak{U}_{1}^{\prime}$ and rk $\mathfrak{V}^{\prime}+\mathrm{rk} \mathfrak{V}^{\prime \prime}=\mathrm{rk} \mathfrak{U}_{1}^{\prime \prime}$. The decomposition (7.11) will be called a refinement of (7.14). In forming $C^{\prime}\left(U_{1}, U_{2}, U_{3}\right)$, we will obtain the fused product of refinements of the decompositions of the involutory centralizers $T\left(C_{\mathfrak{u}_{1^{\prime}}}\left(U_{2}^{\prime}\right)\right)$ and $T\left(C_{\mathfrak{u}_{1^{\prime \prime}}}\left(U_{2}^{\prime \prime}\right)\right)$. Again this will be called a refinement of (7.11). Because we may apply Proposition I.9.1 in forming the decompositions of the involutory centralizers as in (7.11), we may assume that these decompositions are normal decompositions.

Proposition 7.7. Let $S$ be a compatible mutually commuting set of involutions of the first kind. Form the lower centralizer

$$
C^{\prime}(\mathcal{S})=\left(\mathfrak{X}_{1} \times \mathfrak{X}_{2} \times \cdots \times \mathfrak{X}_{r}\right) \circ\left(\mathfrak{X}_{r+1} \times \cdots \times \mathfrak{X}_{t}\right)
$$

where $\mathfrak{X}_{i}, 1 \leqq i \leqq t$, are involutory quasilinear groups. Then for $1 \leqq s<t$,

$$
C^{\prime}(\mathbb{S})=\left(\mathfrak{X}_{1} \times \mathfrak{X}_{2} \times \cdots \times \mathfrak{X}_{s}\right) \circ\left(\mathfrak{X}_{s+1} \times \cdots \times \mathfrak{X}_{t}\right) \text {. }
$$

Proof. The decomposition (7.12) is a refinement of a decomposition (7.14) of the lower centralizer of an involution $U_{i} \in S$ :

$$
C^{\prime}(s)=\mathfrak{u}^{\prime} \circ \mathfrak{u}^{\prime \prime} \text {. }
$$

The direct decompositions in (7.12) are obtained from successive refinements of normal decompositions of involutory centralizers of involutions in $\mathfrak{U}^{\prime}$ and $\mathfrak{U}^{\prime \prime}$. This means that an element $Z \neq 1$ of $Z\left(\mathfrak{u}^{\prime}\right)$, for example, is represented in the direct product $\mathfrak{X}_{1} \times \mathfrak{X}_{2} \times \cdots \times \mathfrak{X}_{r}$ as $Z=Z_{1} \times Z_{2} \times \cdots \times Z_{r}$ where $Z_{i} \neq 1,1 \leqq i \leqq r$. 
Now suppose that $s \leqq r$. The proof in the opposite case will follow similarly. Then we show that

$$
\left(\mathfrak{X}_{s+1} \times \mathfrak{X}_{a+2} \times \cdots \times \mathfrak{X}_{r}\right) \cap\left(\mathfrak{X}_{r+1} \times \cdots \times \mathfrak{X}_{\imath}\right)=1 .
$$

Suppose now that $X=X_{1} \times X_{2} \times \cdots \times X_{\text {, with }} X_{i} \in X_{i}$ is in $X_{0+1} \times X_{0+2}$ $\times \cdots \times X_{t}$. Then $X^{-1}=Y_{s+1} \times Y_{s+2} \times \cdots \times Y_{t}$ with $Y_{i} \in x_{i}$. This implies that $X X^{-1}$ may be written relative to $(7.12)$ as

$$
\left(X_{1} \times \cdots \times X_{\imath} \times Y_{0+1} \times \cdots \times Y_{r}\right) \circ\left(Y_{r+1} \times \cdots \times Y_{t}\right) \text {. }
$$

But then $X_{1} \times \cdots \times Y_{r} \in Z\left(\mathfrak{u}^{\prime}\right) \cap Z\left(\mathfrak{u}^{\prime \prime}\right)$ along with $Y_{r+1} \times \cdots \times Y_{t .}$ Thus $X \in Z\left(\mathfrak{X}_{1} \times \mathfrak{X}_{2} \times \cdots \times \mathfrak{X}_{8}\right) \cap Z\left(\mathfrak{X}_{8+1} \times \mathfrak{X}_{8+2} \times \cdots \times \mathfrak{X}_{t}\right)$. This proves that (7.13) is a fused product.

In the sense of Proposition 7.7, the operations " $O$ " and " $X$ " associate. There will be no ambiguity if we drop parentheses to write, for example,

$$
\begin{aligned}
& \mathfrak{X}_{1} \times \mathfrak{X}_{2} \times \cdots \times \mathfrak{X}_{r} \circ \mathfrak{X}_{r+1} \times \cdots \times \mathfrak{X}_{t} . \\
& \text { III. Proof of Principal Theorem II }
\end{aligned}
$$

8. Proof of theorem. We are at a point where the proof of Principal Theorem I may be adapted to the situation of this paper. This is because our characterization of the lower centralizer of a compatible set of mutually commuting involutions of the first kind now allows us to apply the proofs of the essential theorems and propositions of [4] using the lower centralizer instead of the involutory centralizers. We will make a few remarks explaining certain points of this modification.

First of all, instead of considering involutions in the classes $\Omega_{p}$, we now consider involutions of the first kind which commute with a 1-involution. Whenever we considered commuting involutions, we now consider compatible sets of commuting involutions of the first kind. Rather than consider involutions belonging to the involutory centralizer of a set of mutually commuting involutions in a quasilinear group, we now consider regular involutions of the lower centralizer of a compatible set of mutually commuting involutions of the first kind. As we shall see, these restrictions of compatibility actually do not cause any difficulty in the adaptation of the proofs of the propositions of [4] because we studied involutions which commuted with 1-involutions.

If the lower centralizer of a compatible set of mutually commuting involutions has a decomposition such as (7.12), then we will be able to write a regular involution $X$ belonging to the centralizer as a product of its components $X_{i}$ in the groups $\mathfrak{X}_{i}$ and we may take these components to be involutions. We indicate this by

$$
X=X_{1} \times X_{2} \times \cdots \times X_{r} \circ X_{r+1} \times \cdots \times X_{t} .
$$

We will call (8.1) a representation of $X$ relative to the decomposition (7.12). 
This will take the place of a representation of an involution relative to a particular decomposition of an involutory centralizer. Regular involutions have two representations relative to a decomposition of a lower centralizer, namely (8.1) and

$$
X=-X_{1} \times-X_{2} \times \cdots \times-X_{r} \circ-X_{r+1} \times \cdots \times-X_{\imath}
$$

since $1=-1$ has the decomposition

$$
-1=-1_{1} \times-1_{2} \times \cdots \times-1_{r} \circ-1_{r+1} \times \cdots \times-1_{t}
$$

where $-1_{i}$ is the element of order 2 in $Z\left(\mathfrak{X}_{i}\right)$.

Now we wish to make some specific references to [4]. With regard to Proposition I.4.1, we must make the obvious modification to obtain that every compatible set of mutually commuting involutions is contained in a maximal set of mutually commuting involutions of the first kind which contains $2^{n-1}$ elements. The proof of Lemma I.4.2 needs more than routine revision. We will restate the lemma together with the modified proof.

LEMma II.4.2. Let $\delta$ be a compatible set of mutually commuting involutions of the first kind in the quasiprojective group G. Suppose that there exist two decompositions

$$
\begin{aligned}
& \mathrm{C}^{\prime}(\mathfrak{S})=\mathfrak{X}_{1} \times \mathfrak{X}_{2} \times \cdots \times \mathfrak{X}_{t} \circ \mathfrak{X}_{t+1} \times \mathfrak{X}_{t+2} \times \cdots \times \mathfrak{X}_{\imath}, \\
& C^{\prime}(S)=\mathfrak{V}_{1} \times \mathfrak{Y}_{2} \times \cdots \times \mathfrak{V}_{k} \circ \mathfrak{Y}_{k+1} \times \mathfrak{V}_{k+2} \times \cdots \times \mathfrak{V}_{l}
\end{aligned}
$$

into involutory quasilinear groups. Then $s=l$ and the factors $\mathfrak{X}_{i}$ may be placed in one-to-one correspondence with isomorphic factors $\mathfrak{Y}_{j}$. In particular, the number of components of a given rank is the same in (8.3) as in (8.4).

Proof. Form the group $P\left(E\left(C^{\prime}(s)\right)\right)=P\left(D\left(C^{\prime}(s)\right)\right)$. Just as in the proof of Proposition 6.1, $P\left(E\left(C^{\prime}(\mathcal{S})\right)\right)$ is the direct product of factors $P\left(D\left(\mathfrak{X}_{i}\right)\right)$ in the case of (8.3) or $P\left(D\left(\bigvee_{i}\right)\right)$ in the case of (8.4) which are isomorphic to the groups $\operatorname{PSL}\left(p_{i}, K\right)$ where $p_{i}$ is the rank of $\mathfrak{X}_{i}$ or $\mathfrak{Y}_{i}$. As we remarked in $\S 6$, these components are indecomposable and the Krull-Schmidt Theorem applies. Furthermore, in the present case, the components of rank greater than 1 are determined up to isomorphism by the ranks $p_{i}$ of the corresponding isomorphic groups $P S L\left(p_{i}, K\right)$. From this follows the lemma for the components of rank greater than 1 . Since the sum of the ranks of the components in (8.3) and also in (8.4) is $n$, the component of rank 1 in (8.3) and in (8.4) may be placed in one-to-one correspondence. This proves the lemma.

In our present paper, we have identified the $p$-involutions and $(n-p)$ involutions of $B$ as those involutions in the class $\ell_{p}$. This obviates the necessity of using generalizations of Propositions I.5.3, I.5.4, I.5.5, and I.5.6. In this connection, it should be remarked that the significance of the concept of even involutions is not lost. However, in a quasiprojective group of odd rank, 
an involution is both even and odd since it will be represented as an involution in the center of the two quasilinear decomposition groups, one of which has even rank and the other of which has odd rank. We will still need to speak of even and odd involutions in the quasilinear decomposition groups of the lower centralizer of an involution in the manner of [4]. In particular, Proposition I.6.3 applies exactly as stated for such groups. Propositions I.6.1 and I.6.2 are also stated only for decomposition groups and apply as stated. Propositions I.6.4 and I.6.5 will be applied only to quasilinear decomposition groups.

In $\$ 1.7$, extremal involutions of quasilinear groups are to be interpreted as 1-involutions of quasiprojective groups. In interpreting Proposition I.7.1, remember that the involutions $V$ and $-V$ as well as $U V$ and $-U V$ are to be identified. Actually this will allow a simplification in the proof in the handling of the cases determined by (I.7.10) and (I.7.12). In revising Proposition I.7.5 as in Proposition I.4.1, the orders of the maximal compatible sets of mutually commuting involutions of the first kind will be $2^{n-1}$. Nevertheless, a complete set of mutually commuting 1 -involutions will still contain $n$ elements.

In forming the counterpart of the standard decomposition groups in \$I.8, the distinction between the involutions $U$ and $-U$ in the case of quasiprojective groups becomes artificial. Nevertheless, in any complete set $\Re$ of mutually commuting 1 -involutions $V_{1}, V_{2}, \cdots, V_{n}$ which commute with $U$, we may distinguish two subsets of involutions $\left\{V_{1}, V_{2}, \cdots, V_{p}\right\}$ and $\left\{V_{p+1}, V_{p+2}, \cdots, V_{n}\right\}$ such that $V_{1} V_{2} \cdots V_{p}=V_{p+1} V_{p+2} \cdots V_{n}=U$. All other relations among the commuting involutions $V_{i}$ will be a consequence of these.

The distinction between positive and negative decomposition groups of the lower centralizer of an involution $U$ in the case of quasiprojective groups must be made in an arbitrary manner. If $U$ is an involution of the first kind which is represented as a product of $p$ mutually commuting 1 -involutions, $V_{1}, V_{2}, \cdots, V_{p}$, then it follows from Corollary I.8.4 (modified for quasiprojective groups) that they belong to one of the standard decomposition groups of $C^{\prime}(U)$. Because their product is $U$, they must belong to the same decomposition group. This group will be called the positive standard decomposition group of $C^{\prime}(U)$ relative to the representation $U=V_{1} V_{2} \cdots V_{p}$. In particular, the positive decomposition group will be an involutory quasilinear group of rank $p$. If an involution is given as a $p$-involution, then the positive decomposition group will be taken to be a standard decomposition group of rank $p$. Of course, the other standard decomposition group is to be termed the negative decomposition group.

There are several points in the argument of $\S 1.8$ where our arguments depended upon obtaining a contradiction to exclude certain possibilities. Although it is not possible to obtain these contradictions in our present modi- 
fication of [4], it should be noted that situations will be avoided because of the identification of 1 and -1 .

In adapting Theorem I.8.5 to our present case, one must allow the involutions to be the product of the same number $p 1$-involutions or one to be the product of $p 1$-involutions while the second is the product of $n-p 1$-involutions. Then it may be seen as a consequence of this modification of Theorem I.8.5 that there are $[n / 2]+1$ classes of involutions of the first kind which contain involutions commuting with 1 -involutions. That is, all involutions of the first kind commute with 1 -involutions.

We need also make some remarks about $\S I .13$. In Proposition I.13.1, one must use Axiom B instead of Condition D. In interpreting Proposition I.13.3, let $T(\mathcal{S})$ be the subgroup of $\&$ generated by the involutions of the first kind. Part of the argument of this proposition shows that $\Phi T(\mathbb{S})=\operatorname{PTL}(n, K)$. This is all that is needed. Note that $\Phi$ is an isomorphism of $T(\mathcal{B})$ because of Axiom B. Therefore, $T(\mathbb{B})$ is certainly quasiprojective.

The proof of Principal Theorem II may now be obtained by following the proof of Principal Theorem I.

\section{REFERENCES}

1. J. Dieudonné, On the automorphisms of the classical groups, Memoirs Amer. Math. Soc., no. 2, 1951, pp. 1-95.

2. - La geometrie des groupes classiques, Ergebnisse der Mathematik und ihrer Grenzgebiete, (N. F.), Heft 5, Berlin, Springer-Verlag, 1955.

3. M. Hall, Jr., The theory of groups, New York, Macmillan, 1959.

4. J. H. Walter, On the characterization of linear and projective linear groups. I, Trans. Amer. Math. Soc. vol. 100 (1961) pp. 481-529.

University of Chicago, Chicago, Illinois 